\title{
II. Description et identification
}

\author{
Par Robert Ph. DOLLFUS
}

\section{Description du Plagiorchis \\ obtenu adulte chez des souris blanches de laboratoire}

Corps très allongé longitudinalement, en moyenne 5 fois plus long que large, arrondi antérieurement, atténué postérieurement, gardant à peu près la même largeur dans la plus grande partie de sa longueur. Cuticule spinulée ; la spinulation est serrée antérieurement, elle s'espace peu à peu en direction postérieure et disparaît à un niveau variable, en général peu après le testicule postérieur. La ventouse orale est terminale, à ouverture un peu ventrale. La ventouse ventrale est située à peu près à l'union des deux premiers quarts de la longueur du corps ; son diamètre est un peu supérieur aux $2 / 3$ de celui de la ventouse orale. Il n'y a pas de prépharynx ; le pharynx est globuleux, souvent un peu plus large que long; sa largeur atteint environ la moitié de celle de la ventouse orale. L'œsophage est nul ; les branches intestinales se dirigent latéralement, puis postérieurement et suivent à peu près parallèlement les bords du corps, laissant entre leur bord externe et la paroi du corps un espace large d'environ 1 fois $1 / 2$ à 2 fois leur diamètre; elles se terminent à une petite distance (environ $0,08 \mathrm{~mm}$.) de l'extrémité postérieure du corps. Poche du cirre fortement arquée, contournant le côté droit de la ventouse ventrale, plus large dans ses deux tiers postérieurs, s'amincissant dans son tiers antérieur, qui atteint le pore génital, médian ou submédian, situé presque immédiatement en avant du bord antérieur de la ventouse ventrale. La partie proximale de la poche passe entre la ventouse ventrale et le bord antérointerne de l'ovaire; quelquefois, cependant, elle ne pénètre pas dans cet espace et se trouve contre le bord antéro-externe de l'ovaire. La partie distale de la poche contient le cirre et des cellules prostatiques; la partie proximale, renflée, contient la vésicule séminale interne. Les testicules sont ovales et disposés un peu obliquement l'un par rapport à l'autre dans l'espace intercæcal, touchant l'intestin par leur bord externe. Le milieu de l'antérieur 


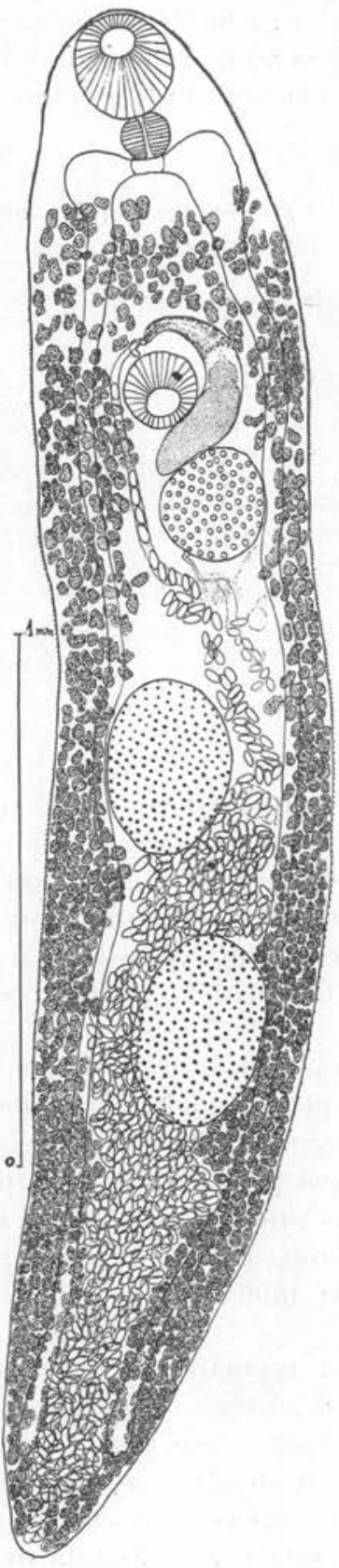

est généralement à peu près au centre du corps. Entre l'ovaire et le testicule antérieur, il y a une distance variable, généralement un peu supérieure au diamètre de l'ovaire, quelquefois un peu inférieure. La distance entre le testicule antérieur et le postérieur est un peu inférieure, ou égale ou un peu supérieure à la mi-longueur du testicule antérieur. Le testicule antérieur est toujours plus petit que le postérieur. L'ovaire, globuleux, plus petit que les testicules, est situé en plus grande partie dans le tiers antérieur du corps et pénètre plus ou moins dans le deuxième tiers; son bord externe est en contact avec le cæcum gauche. Les vitellogènes sont constitués par de nombreux petits follicules de forme irrégulière. Ils peuvent atteindre antérieurement le pharynx et la bifurcation intestinale ou s'étendre un peu moins loin antérieurement, mais forment toujours une large bande transversale d'un bord à l'autre du corps en avant de la ventouse ventrale. Plus en arrière, ils occupent les bords latéraux du corps, jusqu'à l'extrémité postérieure, entourant en grande partie le tube digestif, mais pénétrant peu dans l'espace intercæcal. Un peu en arrière de l'ovaire aboutissent les vitelloductes tranverses et l'oviducte; la glande de Mehlis est un peu à droite de la mi-largeur. Le canal de Laurer est mal distinct chez nos spécimens. Il n'y a pas de receptaculum seminis. L'utérus descendant passe à droite du testicule antérieur, remplit l'espace entre les deux testicules, passe à gauche du testicule postérieur ef s'étend jusqu'à l'extrémité postérieure du corps; ses sinuosités et celles de l'utérus ascendant remplissent presque 
tout l'espace intercæcal en arrière du testicule postérieur. La partie distale de l'utérus ascendant passe contre le bord gauche de la ventouse ventrale pour atteindre le pore génital. Les œufs, extrêmement nombreux, ont une coque mince, ovale et mesurent, en moyenne, $42 \times 22,5 \mu$. La vessie excrétrice est en $\mathrm{Y}$.

Dimensions de quatre adultes à maturité, colorés et montés dans le baume. du Canada après léger aplatissement (en millimètres)

\begin{tabular}{|c|c|c|c|c|}
\hline LONGUEUR & 28 & 2.8 & 2.6 & 26 \\
\hline Largeur.... & 0550 & 0.583 & 0.583 & 0.583 \\
\hline Ventouse orale........ & $0.198 \times 0.187$ & $0.230 \times 0.1 \mathrm{~s} 2$ & $0167 \times 0.177$ & 0.177 \\
\hline Pharynx................ & $0102 \times 0090$ & $0.107 \times 0 \quad 085$ & $0.07 . ; \times 0.102$ & $0.085 \times 0.10 \Sigma$ \\
\hline Ventouse ventrale..... & $0.147 \times 0.147$ & $0.152 \times 0.132$ & $0.122 \times 0.127$ & $0.117 \times 0.137$ \\
\hline Ovaire................. & $0230 \times 0.25$ & $0.230 \times 0.205$ & $0.1<7 \times 0.182$ & $0.187 \times 0.137$ \\
\hline Testicule antérieur... & $0338 \times 0.240$ & $0.356 \times 0.245$ & $0.306 \times 0.225$ & $0230 \times 0.182$ \\
\hline Testicule postérieur... & $0.376 \times 0.249$ & $0.401 \times 0.306$ & $0.356 \times 0.178$ & $0.264 \times 0.215$ \\
\hline
\end{tabular}

\section{Identification et discussion}

Le Plagiorchis ci-dessus décrit appartient à une espèce qui doit être, au moins localement, très commune, la métacercaire ayant été reconnue constamment présente chez les Gammarus du Domaine Universitaire de Richelieu, depuis au moins une vingtaine d'années, pendant l'été. Nous devons toutefois reconnaitre que l'inventaire helminthologique de la faune de Richelieu est encore très fragmentaire.

Les variations individuelles des Plagiorchis sont souvent importantes, au point qu'un spécimen isolé, s'il s'écarte quelque peu de la forme la plus habituelle sous laquelle on connait l'espèce, n'est pas toujours identifiable spécifiquement ; e'est pourquoi beaucoup d'individus un peu aberrants ont été décrits comme de nouvelles espèces. Le nombre des espèces, valables ou non, attribuées par des descripteurs au genre Plagiorchis Max Lühe 1899 (= Lepoderma A. Looss 1899), dépasse actuellement 80.

Plusieurs helminthologistes ont cherché à répartir ces espèces dans des sous-genres ou à distinguer des " groupes d'espèces», mais le but n'a été que partiellement atteint, parce que, chez beaucoup d'espèces, les variations individuelles sont étendues à ce point que, dans un lot de spécimens certainement conspécifiques, trouvés ensemble dans un seul hôte ou dans plusieurs individus du 
même hôte, dans le même biotope, il arrive parfois que quelques individus auraient été placés dans un sous-genre différent ou dans un groupe d'espèces différent s'ils avaient été trouvés isolés (1). Pour délimiter une espèce de Plagiorchis, il faut pouvoir préciser quels caractères moyens, parmi les moins fluctuants, permettent de la définir, en prenant soin d'indiquer quels caractères peuvent être affectés par les variations extrêmes et dans quelles limites (2).

Massino (1927, p. 109-112) a donné une clef de détermination pour 25 espèces de Plagiorchis, où il utilise la position (en tandem ou en oblique) des testicules, les dimensions des testicules comparativement à celles de l'ovaire, l'extension de la poche du cirre en direction postérieure et partiellement l'extension des vitellogènes. Comme, pour un grand nombre d'espèces, ces caractères sont variables chez beaucoup d'individus, ils ne permettent pas de délimiter avec précision des groupes d'espèces.

R. Ed. S. Schulz et A. A. Skvorzov (1931, p. 769) n'ont pas adopté la classification de Massino et ont proposé de prendre en considération la disposition des follicules vitellogènes, cela en désaccord avec Max. Braun (1902, p. 39-40).

Pour Schulz et Skvorzov (1931, p. 771), on peut distinguer deux sous-genres :

(1) Beaucoup d'auteurs ont insisté sur la nécessité d'examiner de nombreux individus d'une même espèce pour pouvoir la délimiter d'espèces voisines. Pour délimiter Plagiorchis arvicola Schulz et Skvorzov 1931, parasite d'Arvicola terrestris (L.), de Pl. proximus F. D. Barker 1915 , parasite d'Ondatra zibethica (L.) et aussi d'A rvicola terrestris (L.) en Tchécoslovaquie, Bozena Erhardova (1958) a examiné comparativement 107 exemplaires provenant d'Arvicola et 212 provenant d'Ondatra. Elle a reconnu que, chez proximus, les dimensions de la ventouse ventrale, des testicules et des autres organes par rapport à la taille des individus étaient proportionnellement bien plus petites que chez arvicola et que le rapport entre la longueur et la largeur du corps ne variait pas entre les mêmes limites chez les deux espèces, étant de $4: 1$ (variant entre 3 à $6: 1$ ) le plus souvent chez proximus et de $2: 1$ (variant entre 1,5 à $2,5: 1$ ) le plus généralement chez arvicolæ. Les caractères affectés par la variation chez ces espèces étaient : 1. La spinulation cuticulaire; celle-ci pouvait s'étendre à toute la surface du corps, ou à une partie seulement, ou pouvait être complètement absente. 2. La longueur de l'œsophage ; chez proximus il arrive qu'il est complètement absent, comme l'avait déjà remarqué J. Grabda $(1954$, p. 21). 3. Le diamètre des ventouses; la ventrale peut être plus grande, plus petite ou égale à l'orale. 4. La grosseur de l'ovaire comparée à celle du pharynx ou de la ventouse ventrale. 5. La position des testicules l'un par rapport à l'autre ; ils peuvent être obliquement, en tandem ou au même niveau. 6. L'extension antérieure des vitellogènes; elle est parfois inconstante, de sorte que des individus de Pl. (Multiglandularis) arvicola Sch. et Skv. pourraient être attribués au sousgenre Plagiorchis.

(2) I. M. Isaïtchikov (1929) a particulièrement étudié les limites de la variation individuelle chez $\mathrm{Pl}$. notabilis W. Nicoll 1909 , tel qu'il a été décrit d'une part par W. Nicoll (1909), d'autre part par V. D. Semenov (1927), le comparant à PI. melanderi Semenov 1927 et à Pl. sveltlakovi I. M. Isaïtchikov 1929. Les différences étant attribuables à des variations individuelles, ces espèces sont en réalité synonymes. 
1) subgen. Plagiorchis, où les vitellogènes ne se rejoignent pas (ou seulement par quelques follicules) en avant de la ventouse ventrale ;

2) subgen. Multiglandularis, où les vitellogènes se réunissent et se confondent nettement en avant de la ventouse ventrale.

Schulz et Skvorzov reconnaissent qu'il y a des intermédiaires entre ces deux dispositions des follicules, mais estiment que, parmi un grand nombre d'individus conspécifiques, ce sont des exceptions. Parmi les 50 espèces qu'ils connaissaient, ils en ont placé 24 dans leur s.gen. Plagiorchis et 19 dans leur s.gen. Multiglandularis; ils en ont laissé sept non classées.

De nombreux auteurs, depuis lors, ont aussi constaté, comme antérieurement Max. Braun, que la coalescence préacétabulaire des vitellogènes pouvait être inconstante chez une même espèce (voir, par exemple : I. Bychovskaja-Pavlovskaja, 1953, fig. 3 ; O. E. Fédorova, 1954 ; St. Furmaga, 1956, p. 583 ; B. Erhardova, 1958, fig. 1 et 6). Cela s'observe, semble-t-il, plus souvent chez les jeunes individus que chez les âgés.

Cependant, dans presque toutes les espèces où ce caractère existe, il est suffisamment net chez la plupart des individus pour que l'on puisse lui reconnaitre une réelle valeur au point de vue systématique ; de plus, chez le plus grand nombre des espèces attribuables à Multiglandularis, peu d'exceptions ont été signalées.

Une telle classification, fondée sur un seul caractère, n'est évidemment pas très satisfaisante; c'est un pis-aller, mais, comme personne n'en a proposé une meilleure, elle a été presque universellement adoptée et il est incontestable qu'elle rend les plus grands services.

La clef de détermination des Plagiorchis, donnée par $O$. Wilford Olsen (1937, p. 325-335, fig. $50 \mathrm{~A}-77$ ), s'applique à 23 espèces et deux variétés pour le s. g. Plagiorchis, 20 espèces pour le s. g. Multiglandularis. Faute de renseignements, quatre espèces (décrites par des helminthologistes russes) n'ont pas été comprises dans la clef ; 28 espèces sont figurées.

K. I. Skrjabin et D. N. Antipin (1958, p. 99-343, fig. 15-97) ont donné les descriptions et figures de 40 espèces et une variété pour le sous-genre Plagiorchis et de 37 espèces et une variété pour le sous-genre Multiglandularis.

Cette publication de Skrjabin et Antipin permet de prendre connaissance simultanément et comparativement des descriptions et figures de beaucoup d'espèces décrites et figurées dans des périodiques russes, pour la plupart inaccessibles en dehors de l'U.R.S.S. 
Toutes les espèces décrites et figurées dans l'ouvrage de Skrjabin et Antipin sont-elles valables? Lesquelles sont synonymes ? Skrjabin et Antipin n'indiquent quelques synonymes que pour un très petit nombre d'espèces. Ils semblent n'avoir pas tenu compte des synonymies très étendues proposées par O. E. Fédorova (1954). Cet auteur, parmi les 62 espèces ou sous-espèces restées dans le genre après les éliminations, en a réduit le nombre à 36 (33 espèces +3 sous-espèces) par mises en synonymie. Malheureusement, le travail de Fédorova est resté inédit et nous n'en connaissons qu'un résumé (3). Ce résumé a été utilisé par Klaus Odening (1959), à qui l'on doit la plus récente révision du genre (4).

$K$. Odening a essayé de rendre le genre plus homogène et en a donné une nouvelle définition (p. 16). Il en a éliminé les espèces chez lesquelles l'utérus ne s'étend jamais en arrière du testicule postérieur et les a transférées, conformément à ce que j'avais moimême déjà proposé $(1949$, p. 440 , note 1$)$, dans la sous-famille Opisthioglyphinæ R.-Ph. D. 1949. Sont ainsi éliminées de Plagiorchis des espèces telles que fastuosus L. Szidat $1924=$ morosovi A. Sobolev 1946, arcuatus Strom 1924, mustelæ Petrov et Kadenazi 1954, toutes les formes tombant en synonymie d'Opisthioglyphe (Rubenstrema) exasperatum (Rudolphi 1819) (5), dont Plagiorchis microti A. Soltys 1949 et formes voisines, telles que Plagiorchis opisthovitellinus A. Soltys 1954 .

En outre, Odening (p. 16-17) élimine de Plagiorchis les espèces de Plagiorchïinæ où les vitellogènes ne s'étendent pas jusqu'à l'extrémité postérieure du corps, l'utérus remplissant seul cette extrémité (6). Sont ainsi éliminées les espèces didelphidis C. Parona 1894, ramlianum A. Looss 1896, himalayi Jordan 1930, momplei R.-Ph. Dollfus 1932, lenti Freitas 1941, yoshidensis T. Ogata 1942, hepaticus A. Lutz 1928, molini Lent et Freitas 1945, ptschelkini A. Sobolev 1946 (7).

(3) Le résumé d'O. E. Fedorova a paru en 1954, mais s'arrête à l'année 1953 , c'est pourquoi il n'y est pas question de Pl. (Pl.) blatnensis J. Chalupsky (1954, p. 181-188, fig. 1-2) de l'intestin grêle de Microtus arvalis Pallas, de Blatna (Tchécoslovaquie) et de quelques autres espèces de Plagiorchis.

(4) Il y a quelques omissions dans la liste des espèces donnée par Odening, par exemple Pl. pholkeewi V. J. Panin 1956, de Mus musculus L.; en outre, depuis le temps où Odening a rédigé sa révision, de nouvelles espèces ont été publiées. En 1958 ont été déerits et figurés $P l$. (Pl.) raabei S. Furmaga 1956 et P. (M.) stefanskii S. Furmaga 1956 .

(5) Pl. exasperatum (Rud. 1819) avait été éliminé du genre Plagiorchis par O. E. Fedorova $(1954$, p. 11) en même temps que Pl. ramlianus Looss 1899 et Fl. corfi Lamont 1921.

(6) Comme cela a lieu, par exemple, chez Astiotrema A. Looss 1900.

(7) Aucune de ces espèces, sauf ptschelkini, ne se trouve dans la liste des 3 ? $^{\circ}$ retenues dans le genre par 0 . E. Fedorova (1954, p. 11-12). 
Ces éliminations sont peut-ètre, en partie, contestables (8).

Dans le sous-genre Plagiorchis, quatre groupes d'espèces ont été distingués par Odening (1949, p. 17-18) :

a) groupe vespertilionis : quatre espèces et deux sous-espèces ;

b) groupe maculosus : une espèce et deux sous-espèces ;

c) groupe elegans-triangularis : quatre espèces et deux sousespèces ;

d) espèces n'entrant pas dans les groupes précédents: quatre espèces.

Dans le sous-genre Multiglandularis, trois groupes d'espèces :

a) groupe cirratus-laricola: six espèces et trois sous-espèces ;

b) groupe multiglandularis : une espèce et deux sous-espèces ;

c) groupe notabilis-muris : quatre espèces et quatre sous-espèces.

Faute de renseignements suffisants, 10 espèces n'ont pas été admises par Odening dans les groupes précédents.

$$
* *
$$

Les spécimens du Plagiorchis obtenu par nous expérimentalement, chez des souris blanches de laboratoire, appartiennent évidemment au sous-genre Multiglandularis et au groupe cirratuslaricola qui comprend, d'après Odening (1949, p. 18) :

1. P. (M.) cirratus cirratus (Rudolphi 1802); synon. P. brauni Massino 1927, P. massino Petrov et Tichonov 1927, P. loossi Massino 1927, P. casarcii (Mehra 1937).

2. P. (M.) cirratus potanii (Skrjabin 1928).

3. P. (M.) skrjabini (Massino 1927).

4. P. (M.) schuchovi (Isaïtchikov 1920).

5. P. (M.) asper (M. Stossich 1904).

6. P. (M.) mentulatus (Rudolphi 1819).

7. P. (M.) laricola laricola (Skrjabin 1924).

8. P. (M.) laricola mutationis (Panova 1927).

9. P. (M.) laricola ferruginum (Mehra 1927).

Nous avons comparé nos spécimens à ces espèces et sous-espèces, utilisant les figures originales ou, à défaut, les copies publiées par Skrjabin et Antipin. La seule espèce qui corresponde exactement est cirratus, sous sa forme typique cirratus : même habitus, mêmes dimensions, même position des testicules par rapport à l'ovaire et

(8) Par exemple, l'élimination de Pl. mompléi (R.-Ph. Dollfus 1932). 
à l'extrémité postérieure du corps. La comparaison avec les espèces synonymes est aussi concordante.

Cette espèce a été souvent signalée ; elle parasite un grand nombre d'espèces d'Oiseaux d'Europe et d'Asie Occidentale ; cependant, elle n'a pas été souvent figurée sous le nom spécifique cirratus.

La figure originale de cirratus, donnée par Rudolphi (1808, pl. VI, fig. 7), d'après un spécimen récolté chez Corvus monedula L., à Greifswald (Saxe), est peu caractéristique, et Max. Braun (1902, p. 43-44), qui a redécrit l'espèce d'après les spécimens de Rudolphi, n'a donné aucune figure. Une bonne description et de bonnes figures de cirratus ont été données par Paul Mühling (1897, p. 262-266, pI. XVII, fig. 5 ; pl. XVIII, fig. 12), d'après des spécimens récoltés par Max. Braun à Königsberg chez Corvus corone L. Pour les œufs, Mühling indique 0,0375-0,04 $\times 0,0187 \mathrm{~mm}$., Max. Braun (1902, p. 44$), 0,0237-0,035 \times 0,0182-0,0228 \mathrm{~mm}$.

O. E. Fédorova (1954) a considéré comme synonymes de cirratus : brauni Massino 1927, loossi Massino 1927, massino Petrov et Tichonov 1927 et même $P$. uhlwormi Massino 1927. Pour uhlwormi, dont les vitellogènes se rapprochent sans se rejoindre en avant de la ventouse ventrale, K. Odening (1959, p. 19) n'est pas d'accord, mais il ajoute un synonyme de plus : Lepoderma casarcii Mehra 1937. Ces synonymies ont permis d'étendre considérablement la liste des hôtes de l'espèce ; en Eurasie, les hôtes appartiennent aux Corvidæ, Motacillidæ, Sturnidæ, Fringillidæ, Laridæ, Falconidæ, Anatidx ; à des hôtes Oiseaux ont été ajoutés, comme hôtes accidentels, la souris blanche, le chien et le chat domestiques.

Une nouvelle description de cirratus a été donnée par K. Odening (1959, p. 19-21, fig. 1-2), d'après des exemplaires de divers âges qu'il trouva à l'Université d'Iéna dans l'intestin grêle de souris blanches de laboratoire. Pour les œufs, il a mesuré 0,033-0,043 $\times$ 0,017-0,028 mm., ce qui est normal pour l'espèce et s'accorde avec nos mesures $(42 \times 22,5 \mu)$. A cirratus, mais avec un point de doute, Odening a aussi rapporté de jeunes spécimens d'une poule domestique d'une ferme des environs d'Iéna.

A Richelieu (Indre-et-Loire), dans la nature, cirratus n'a pas, jusqu'à présent, été rencontré, mais l'inventaire helminthologique de Richelieu n'est encore qu'à son début. Il est vraisemblable que plusieurs espèces d'Oiseaux, sédentaires ou de passage à Richelieu, sont hôte définitif de cirratus. Plusieurs d'entre elles, peut-être, s'alimentent de Gammarus, mais il n'est pas actuellement démontré que les Gammarus sont des hôtes permettant la continuation du cycle évolutif dans la nature. Il est possible que la cercaire ne 
s'enkyste pas seulement dans des Gammarus, mais s'enkyste aussi dans des larves aquatiques d'insectes (9), qui, devenus adultes, sont mangés par l'Oiseau hôte définitif où le Distome pourra acquérir sa maturité génitale.

\section{OUVRages CITÉS}

ANGel (Madeline), 1959. - An account of Plagiorchis maculosus (Rud.), its synonymy and its life history in South Australia. Transact. Royal Soc. South Australia, vol. LXXXII (1959), p. 265-281, fig. 1-10 et tableau hors-texte.

braun (Maximilian), 1902, - Fascioliden der Vögel. Zoolog. Jahrbücher. System., Bd XVI, Heft 1, 6-5-1902, p. 1-162, pl. I-VIII, fig. 1-99.

Bychovskaja-Parlovskaja (Irène), 1953. - Faune des Trématodes d'Oiseaux de la Sibérie occidentale et sa dynamique. Magasin de Parasitologie de l'Institut Zoologique de l'Académie des Sciences de l'U.R.S.S., tome XV, 1953 , p. 5-116, fig. 1-67b.

Chalupsiy (Josef), 1954. - Plagiorchis blatnensis n. sp. (Plagiorchiidæ, Trematoda) from the small intestine of Microtus arvalis Pall. Acta Societatis Zoologice Bohemoslovenice, vol. XVIII, (1954), n 3 , p. 181-188, fig. 1-2.

Dollfy's (Robert-Ph.), 1949. - Distoma rubens F. Dujardin 1845 (= exasperatum Rudolphi 1819), retrouvé en France et redécrit. Annales de Parasitologie hum. et compar., t. XXIV, n 5-6, déc. 1949, p. 436-442, fig. 1-5.

Erнardova (Bozena), 1958. - Morphologische Veränderlichkeit des Drehwurmes Plagiorchis arvicola Schulz et Skworzov 1931 und Pl. proximus Barker 1915. Acta Societatis Zoologica Bohemoslovenice, t. XXII, n 3,1958 , p. 266-278, fig. 1-8.

Fedorova (O. E.), 1954. - La variabilité des caractères morphologiques et leur importance pour la systématique des Trématodes du genre Plagiorchis Lühe 1899. Résumé d'auteur d'une dissertation présentée pour la candidature en Sciences biologiques. Université de Léningrad, 1954, $12+1 \mathrm{p}$.

(9) L'enkystement expérimental des cercaires de Plagiorchis a été plus souvent observé chez des larves d'insectes que chez des Crustacés. W. Nöller et K. Ullrich (1927), pour obtenir la métacercaire de Pl. maculosus (Rud.), ont utilisé des larves de Chironomides. Karl Strenzke (1952), pour la même espèce de Plagiorchis, a obtênu l'enkystement dans des larves de Chaoborus crystallinus (Deg.), Chironomus thummi Kieff., Psectrotanypus varius (Fabr.), difficilement dans celles de Culex pipiens L., mais pas chez Daphnia, et une larve de Coléoptère.

Madeline Angel (1959), avec la cercaire du Plagiorchis (P. clelandi S. J. Johnston $1917=P$. spatulatus S. J. Johnston 1917) qu'elle identifie à $P$. maculosus (Rud.), a obtenu l'enkystement dans des larves de moustiques, mais pas dans des crustacés (Daphnia, Paratya, Cherax, Chiltonia) ni dans divers autres hôtes. Elle a constaté qu'une cercaire voisine, celle de $P l$. jaenschi T. H. Johnston et M. Angel 1951, pénètre et s'enkyste rapidement chez le crustacé Cherax destructor Clark 1936 [Décapode Parastacide], qu'elle considère comme l'hôte intermédiaire normal de l'espèce. 
Furmaga (Stefan), 1956. - The helminth fauna of field rodents (Rodentia) of the Lublin environment. Acta Parasitologica Polonica, Varsovie, t. XV, fasc. 2, p. 9-50, fig. 1-16c.

Grabda (Jadwiga), 1954. - Les parasites internes du rat musqué - Ondatra zibethica (L.) - des environs de Bydgoszaz (Pologne). Acta Parasitologica Polonica, t. II, n. 2, 1954, p. 17-38, fig. 1-6.

Isaïtchikov (Isaac M.), 1929. - Au sujet des limites de la variation individuelle des espèces du genre Plagiorchis Lühe 1899. Trudy Sibirsk. Veterin. Instituta, t. X, 1929 , p. $275-283$.

Massino (B. G.), 1927. - Die Bestimmung der Arten der Gattung Plagiorchis Lühe. Sammlung helminthologischer Arbeiten Prof. Dr. K. I. Skrjabin von seinen Schülern gewidmet. Moscou, 1927, p. 108-113, fig. 1-6.

Mühlivg (Paul), 1897. - Beiträge zur Kenntniss der Trematoden. Archiv für Naturgeschichte, Berlin, Jahrg. LXII (1896), Bd I, Heft 3, Febr. 1897, p. 243-279, pl. XVII-XIX, fig. 1-17.

ODEning (Klaus), 1959. - Ueber Plagiorchis, Omphalometra und Allocreadium (Trematoda, Digenea). Zeitschrift für Parasitenkunde, Bd XIX, Heft 1, abgeschlossen 17-3-1959, p. 14-34, fig. 1-7.

NöLler (Wilhelm) et Ulllrich (Konrad), 1927. - Die Entwicklung einer Plagiorchis-Art (Ein Beitrag zur Kenntnis der Cercariæ armatæ). Sitzungsber. d. Gesellschaft naturforsch. Freunde, 1-11-1927, p. 81-96, pl. I, fig. 1-26.

OLsen (O. Wilford), 1937. - A systematic study of the Trematode subfamily Plagiorchiine Pratt, 1902. Transact. Amer. Microscop. Soc., vol. LVI, $\mathrm{n}^{\circ} 3$, july 1937 . p. 311-339, pl. I-V, fig. 1-80.

RUdol.PH (Carl, Asmund), 1808. - Entozoorum sive vermium intestinalium historia naturalis, vol. I, Amstelædami 1808 , xxvI +527 p., pl. I-VI.

Schulz (Richard Ed. S.) et Skvontzov (A. A.), 1931. - Plagiorchis arvicole n. sp. aus der Wasserratte. Zeitschr. für Parasitenkunde, Bd III, Heft 4, abgeschl. 28-8-1931, p. 765-774, fig. 1-3.

Semenov (Vasili, Dmitriévicin), 1917. - Vogeltrematoden des westlichen Bereiches der Union S.S.R. Sammlung helminthologischer Arbeiten Prof. Dr. K. I. Skrjabin von seinen Schülern gewidmet. Moskau, p. 221-271, fig. $1-10+2$ tableaux hors-texte.

SkRJabin (Konstantin I.) et ANTIPIN (D. N.), 1958. - Superfamille Plagiorchioidea Dollfus 1930. In K. I. SKrJabin: Trématodes des animaux et de l'homme, tome XIV, Edition de l'Académie des Sciences de l'U.R.S.S., Moscou, 1958 , p. $75-666$, fig. $15 a-185 b$.

Strenzke (Karl), 1952. - Der Wirtswechsel von Plagiorchis maculosus. Keitschrift für Parasitenkunde, Berlin, Bd XV, Heft 5, abgeschiossen 2-12-1952, p. 369-391, fig. 1-5.

Strenzle (Karl), 1953. - Zuckmücken als Zwischenwirte für Saugwürmer. Mikrokosmos, Bd XLII, n. 8, 1953, p. 169-174, fig. 1-10.

Pour une bibliographie exhaustive des Plagiorchis, on se reportera principalement à SkrJabin et ANTipin (1958) et Odening (1959). 